University of Nebraska - Lincoln

DigitalCommons@University of Nebraska - Lincoln

Ralph Skomski Publications

Research Papers in Physics and Astronomy

2009

\title{
Magnetic Correlations in Nanocomposite FePt:Au and FePt:C Films
}

Thomas A. George

University of Nebraska-Lincoln, tgeorge1@unl.edu

Ralph Skomski

University of Nebraska-Lincoln, rskomski2@unl.edu

David J. Sellmyer

University of Nebraska-Lincoln, dsellmyer@unl.edu

Follow this and additional works at: https://digitalcommons.unl.edu/physicsskomski

Part of the Physics Commons

George, Thomas A.; Skomski, Ralph; and Sellmyer, David J., "Magnetic Correlations in Nanocomposite FePt:Au and FePt:C Films" (2009). Ralph Skomski Publications. 58.

https://digitalcommons.unl.edu/physicsskomski/58

This Article is brought to you for free and open access by the Research Papers in Physics and Astronomy at DigitalCommons@University of Nebraska - Lincoln. It has been accepted for inclusion in Ralph Skomski Publications by an authorized administrator of DigitalCommons@University of Nebraska - Lincoln. 


\title{
Magnetic correlations in nanocomposite FePt:Au and FePt:C films
}

\author{
T. A. George, ${ }^{\text {a) }}$ R. Skomski, and D. J. Sellmyer \\ Department of Physics and Astronomy and Nebraska Center for Materials and Nanoscience, \\ University of Nebraska, Lincoln, Nebraska 68588, USA
}

(Presented 12 November 2008; received 17 September 2008; accepted 27 November 2008; published online 27 March 2009)

\begin{abstract}
The nanostructure and magnetism of $L 1_{0}$-ordered FePt crystallites in matrices of $\mathrm{Au}$ and $\mathrm{C}$ are investigated as a function of the $\mathrm{Au}$ and $\mathrm{C}$ contents. Emphasis is on the relation between hysteresis-loop shape, magnetic correlation length, and structural disorder, as relevant to magnetic recording media. The highly (001) textured thin films are produced by annealing $[\mathrm{Fe} / \mathrm{Pt} / X]_{n}$ multilayers. The coercivity and the hysteresis-loop slope at coercivity increase and decrease, respectively, with increasing volume fraction of the matrix. The magnetic force microscopy images show that the slope decrease is accompanied by a reduction in the magnetic correlation length. These changes are due to the reduced intergranular exchange coupling, and a simple model describes how interparticle exchange cooperatively increases both the loop slope and the correlation length. (C) 2009 American Institute of Physics. [DOI: 10.1063/1.3073653]
\end{abstract}

\section{INTRODUCTION}

$L 1_{0}$-ordered FePt recently has attracted much attention as a material for ultrahigh-density perpendicular magnetic recording (areal density $>1 \mathrm{~Tb} /$ in. $^{2}$ ). The magnetocrystalline anisotropy of this hard-magnetic phase is around $7 \mathrm{MJ} / \mathrm{m}^{3}\left(7 \times 10^{7} \mathrm{ergs} / \mathrm{cm}^{3}\right)$, second only to the more corrosive rare-earth intermetallics. ${ }^{1,2}$ However, FePt deposited at ambient temperatures is found in the fcc disordered phase and tends to have a random distribution of the (001) crystalline axis. This is unsuitable for perpendicular media, as the ordered $L 1_{0}$ [001] easy axis is required to be aligned normal to the film surface. Even after ordering by heat treatment, these films typically have large, highly exchange-coupled grains which greatly increase noise and limit the achievable areal bit density. ${ }^{3-5}$ Controlling the easy-axis orientation and intergranular interactions and understanding how these are affected by the real structure are necessary steps to realize FePt as a recording medium.

The control of the $L 1_{0}$ easy-axis orientation is achievable in magnetron sputtered thin films through various deposition and/or processing techniques. ${ }^{5-8}$ Grain size and intergranular interactions have been controlled by the addition of a nonmagnetic material, such as $\mathrm{MgO}, \mathrm{Ag}, \mathrm{Au}, \mathrm{C}$, or $\mathrm{AlO}_{x}$, as a decoupling and dispersing matrix. ${ }^{9-13}$ The interactions can be analyzed directly by magnetic force microscopy (MFM) or indirectly through the shape of the hysteresis loop. In particular, the slope at coercivity of a hysteresis curve, $\alpha$ $=(4 \pi d M / d H)_{H c}$, may affect the performance of a magnetic recording medium. ${ }^{3}$ Some attention has been paid to the relationship between the parameter $\alpha$ and the magnetic correlation length, $L_{m}$, as measured by MFM, ${ }^{10,14}$ but little work has been done to connect the two with a model to gain information about the real structure of a system.

The correlation length $L_{m}$ of a macroscopically homogeneous and isotropic magnet is defined in terms of the corre-

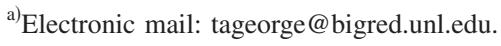

lation function, $C(R)$ which is experimentally accessible by neutron or x-ray scattering where $C(R) \sim 0$ for $|\mathbf{R}| \sim L_{m}$. Many systems are reasonably well described by $C(R)$ $=C(0) \exp \left(-R / L_{m}\right) .{ }^{15-18}$ Our emphasis here is on correlations between neighboring grains, that is, on interaction domains.

In this paper, we report experimental results of two nanocomposite systems, FePt:Au and FePt:C. These two matrix materials have been shown to magnetically decouple the FePt grains while maintaining a preferred (001) texture. ${ }^{10,19}$ Particular attention is paid here to the properties $\alpha$ and $L_{m}$ as functions of matrix content. A model is proposed to explain the close correspondence between the two parameters.

\section{EXPERIMENTAL METHODS}

All films were made by magnetron sputtering with base pressures of about $7 \times 10^{-7}$ Torr. Details of the FePt:Au films can be found in Ref. 19. The films are initially deposited as multilayers with the structure $[\mathrm{Fe} / \mathrm{Pt} / X]_{n}$ with individual layer thicknesses ranging from about 0.1 to $1 \mathrm{~nm} .^{20}$ The FePt:C films were deposited as a repeated multilayer structure on thermally oxidized silicon substrates at ambient temperature from $99.99 \%$ pure $\mathrm{Fe}, \mathrm{Pt}$, and $\mathrm{C}$ elemental targets with 5.1 mTorr of Ar working gas. The volume percentage of carbon was controlled by its deposited layer thickness and ranged from 0 to $38.6 \mathrm{vol} \%$. To form the $L 1_{0}$ phase, microstructure, and (001) texture, postdeposition annealing was performed in a rapid-thermal-annealing oven at $600-700{ }^{\circ} \mathrm{C}$ for $5 \mathrm{~min}$ in a $5 \% \mathrm{H}_{2}$ in Ar forming gas. Crystalline phase and texture were confirmed by x-ray diffraction and hysteresis loops were obtained at room temperature with a superconducting quantum interference device magnetometer in fields up to $7 \mathrm{~T}$.

Magnetic correlation lengths were estimated using a Dimension 3100 MFM with a NanoScope III SPM controller using interleave mode with a constant lift height of $15 \mathrm{~nm}$. All the FePt: $X$ samples were measured in their virgin state. The raw images were processed (i.e., flattened if necessary) 


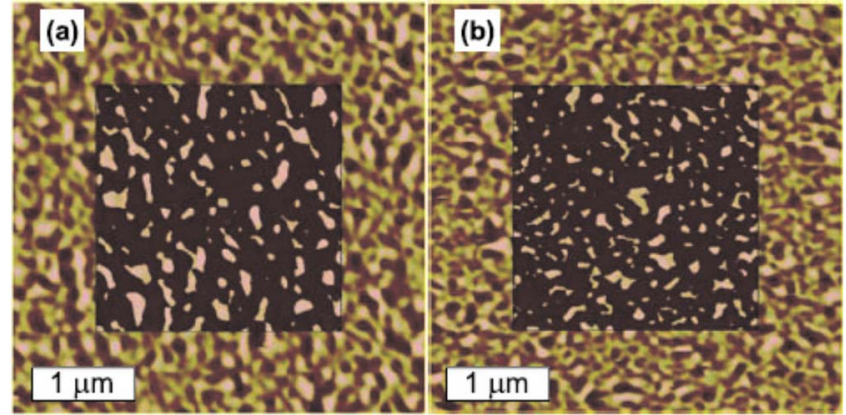

FIG. 1. (Color online) MFM images for FePt:C with (a) 0 and (b) 33 vol \% C. The inner squares are filtered for features greater than $50 \%$ of the maximum signal.

using the NanoScope III software to extract a value for $L_{m}$. The center regions of each scan were filtered for features greater than $50 \%$ of the maximum signal. Grain-size-analysis software determined the mean feature size in this region and the square root of this value was taken as $L_{m}$. A correction function of the form $L_{m}^{2}+L_{\min }^{2}=L_{\exp }^{2}$ (where $L_{\min }$ is the smallest resolvable feature size due to tip quality and lift height and was taken to be $30 \mathrm{~nm}$ ) was applied to deconvolute the raw data.

\section{RESULTS}

Figure 1 shows the MFM scans for the FePt:C system with 0 and 33.3 vol \% C. These scans illustrate how the magnetic domains decrease in size with increasing matrix content. Without carbon, $L_{m}$ was $80.4 \mathrm{~nm}$. This decreased to $50.2 \mathrm{~nm}$ for 38.6 vol \% carbon. Figure 2 shows how $L_{m}$ varies with matrix content for both systems. For the FePt:Au, $L_{m}$ ranges from $90 \mathrm{~nm}$ for gold-free films to $73.8 \mathrm{~nm}$ for 32.4 vol \% Au.

The hysteresis-loop slope at coercivity is also related to intergranular exchange, usually increasing with exchange and decreasing with matrix content. In the FePt:C system, Fig. 2(a), $\alpha$ decreases from 4 for carbon-free films to 0.8 for 38.5 vol \% C. Figure 2(b) shows a similar trend for the FePt:Au system; parameter $\alpha$ decreases from 5.7 to 0.9 as the $\mathrm{Au}$ vol \% is increased. Features such as switching-field distribution, magnetostatic interactions, particle geometry, and the electronic properties of the matrix also affect loop slope. Consequently, exchange strength cannot be derived from a single value of $\alpha$. However, trends in $\alpha$ are indicative of trends in exchange, i.e., exchange between grains increases as the slope becomes steeper. In practice, the exchange interactions are often of the contact type, that is, grains are relatively strongly coupled if they touch each other. In addition, there is some exchange mediated by grain boundaries or by the matrix, where the coupling is reduced ${ }^{18}$ and the magnetization reversal becomes reminiscent of pinning. ${ }^{21}$ In fact, seemingly minor real-structure changes may translate into very different coupling and reversal mechanisms and yield significantly different parameters in micromagnetic models.

It is clear from Fig. 2 that $L_{m}$ and $\alpha$ are closely connected; both reflect intergranular exchange and are controlled by the volume fraction of the matrix phase. It is well known that the slope $\alpha_{0}$ of an ensemble of noninteracting particles is
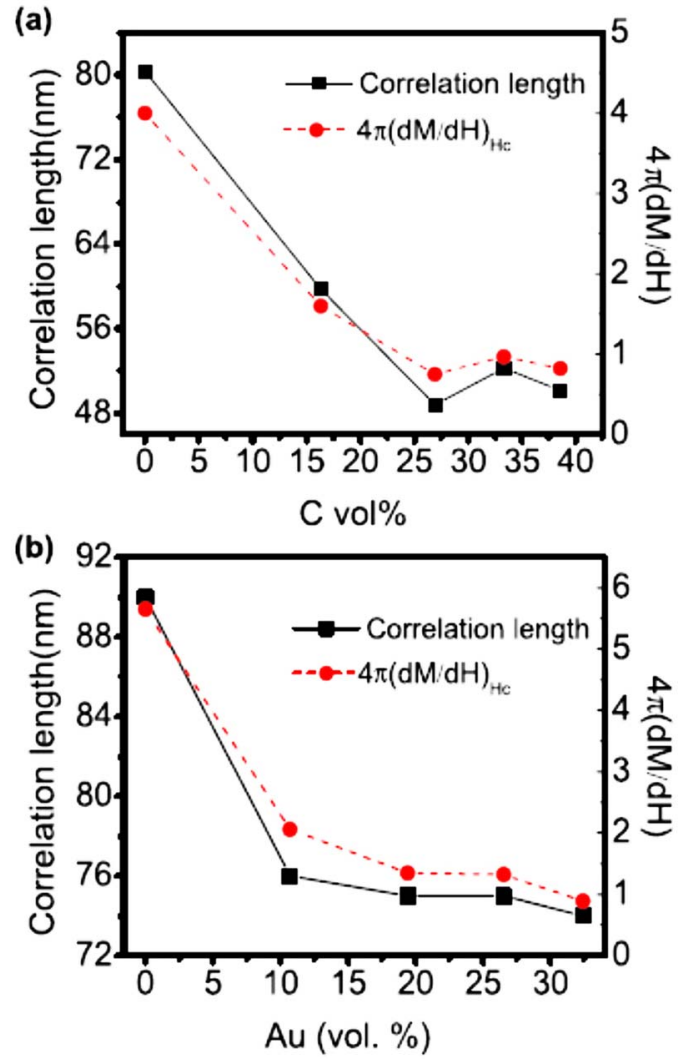

FIG. 2. (Color online) Correlation lengths and parameter $\alpha$ for (a) FePt:C and (b) FePt:Au.

determined by the particles' switching-field distribution, $\alpha_{0}$ $\sim M_{s} / \Delta H_{\text {sw }}$. Adding an exchange and/or magnetostatic interaction field $H_{\text {int }}$ to the external magnetic field yields for small interactions ${ }^{10}$

$$
\frac{\alpha}{\alpha_{0}}=1+\alpha_{0} \frac{H_{\text {int }}}{M_{s}} .
$$

If two noninteracting particles of volume $V_{0}$ touch each other with a probability $W$, then the average volume of the cooperative units increases to $V=V_{0}(1+W)$. In this equation, $W$ can be expressed as $W=\alpha_{0} \Delta H_{\text {int }} / M_{s}$, where $\Delta H_{\text {int }}$ is a net interaction field. Assuming that each particle is coordinated by $z$ neighbors, we must take into account that we have been describing the clustering of two particles only, because the other $z-1$ neighbors have in general very different switching fields. In other words, the interaction cooperatively couples ${ }^{22}$ pairs with similar switching fields, and the remaining $z-1$ neighbors do not interfere in the lowest order. This means that $\Delta H_{\mathrm{int}}=H_{\mathrm{int}} / z$, and for small $H_{\mathrm{int}}$, we have

$$
\frac{V}{V_{0}}=\left(1+\alpha_{0} \frac{H_{\mathrm{int}}}{M_{s}}\right)^{1 / z} \text {. }
$$

Comparing Eqs. (1) and (2) yields, with $V \sim L_{m}^{3}$,

$$
\frac{L_{m}}{L_{0}}=\left(\frac{\alpha}{\alpha_{0}}\right)^{1 / 3 z} \text {. }
$$

Equation (3) makes it possible to extract real-structure information from $L_{m}$ and $\alpha$. 


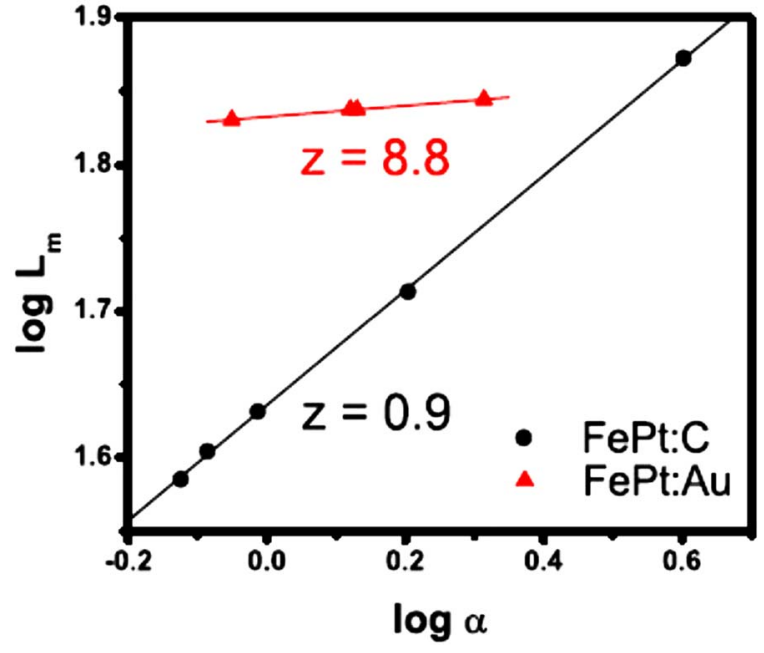

FIG. 3. (Color online) Relationship between correlation length $L_{m}$ and loop slope $\alpha$. This double-logarithmic plot is used to determine the parameter $z$.

Figure 3 shows a plot of $\log \left(L_{m}\right)$ versus $\log (\alpha)$ for both systems. The microstructural parameters $z \approx 0.9$ for FePt:C and $z \approx 8.8$ for FePt:Au are very different from each other. The latter is consistent with a picture of a three-dimensional random network of particles. The former is relatively small and may indicate a reversal mechanism different from that assumed in Eqs. (1)-(3). A more thorough explanation of $z$ requires an explicit calculation of $\alpha$ and $L_{m}$ from the coupling properties of the matrix and from the structural disorder of the granular system.

\section{DISCUSSION AND CONCLUSIONS}

We have assumed that the correlation length, or the size of the interaction domains, is governed by two-particle interactions. Physically, the interactions are not short range but propagate through the magnet, and the MFM pictures correspond to the result of this propagation. As a rule, the correlation function $C(R)$ exhibits a strong dependence on the applied magnetic field. For infinite fields $\mathbf{H}=H_{z} \mathbf{e}_{z}, \mathbf{M}(\mathbf{r})$ $=M_{s} \mathbf{e}_{z}$, and $C(R)=0$. For strong but finite fields, there is a small perpendicular magnetization component $m$, leading to $C(\mathbf{r})=M_{s}^{2}\langle\mathbf{m}(\mathbf{r}) \cdot \mathbf{m}(\mathbf{r}+\mathbf{R})\rangle$, where $\mathbf{m}(\mathbf{r})$ can be determined by methods described elsewhere. ${ }^{22,23}$ The situation is often more complicated in that fields used in typical experiments are homogeneous on the nanoscale, in contrast to actual write fields, which are localized on a length scale $D$ given by the head size and the head-to-medium distance. This localization would reduce the measured correlation length to a value that depends on the type of disorder present in the system, but varies between $L_{m}$ ( $D=$ infinity) and roughly the Bloch-wall width $(D=0)$.
In conclusion, we have investigated granular FePt:C and FePt:Au thin films with potential for perpendicular magnetic recording. The films are $L 1_{0}$ ordered with perpendicular $c$-axis orientation, but intergranular exchange affects the performance of the material. The relationship between the hysteresis-loop slope $\alpha$ and the magnetic correlation length $L_{m}$ at coercivity is governed by a real-structure dependent parameter $z$. The studies here correlating $L_{m}$ and $\alpha$, when extended to noise measurements in magnetic media, may enable useful comparisons of competing films structures and compositions as low-noise recording media.

\section{ACKNOWLEDGMENTS}

The authors are grateful for financial support from NSFMRSEC (Contract No. DMR-0213808), INSIC (T.A.G. and D.J.S.), DOE (Contract No. DE-FG02-04ER46152) and NCMN (R.S.).

${ }^{1}$ O. A. Ivanov, L. V. Solina, and V. A. Demshina, Phys. Met. Metallogr. 35, 81 (1973).

${ }^{2}$ T. Klemmer, D. Hoydick, H. Okumura, B. Zhang, and W. A. Soffa, Scr. Metall. Mater. 33, 1793 (1995).

${ }^{3}$ N. Honda, K. Ouchi, and S. Iwasaki, IEEE Trans. Magn. 38, 1615 (2002). ${ }^{4}$ J. J. Miles, D. M. McKirdy, R. W. Chantrell, and R. Wood, IEEE Trans. Magn. 39, 1876 (2003).

${ }^{5}$ Y. F. Xu, M. L. Yan, and D. J. Sellmyer, J. Nanosci. Nanotechnol. 7, 206 (2007).

${ }^{6}$ M. L. Yan, N. Powers, and D. J. Sellmyer, J. Appl. Phys. 93, 8292 (2003). ${ }^{7}$ S. Jeong, Y. N. Hsu, D. E. Laughlin, and M. E. McHenry, IEEE Trans. Magn. 36, 2336 (2000).

${ }^{8}$ Y. F. Xu, J. S. Chen, and J. P. Wang, Appl. Phys. Lett. 80, 3325 (2002).

${ }^{9}$ T. Suzuki and K. Oushi, IEEE Trans. Magn. 37, 1283 (2001).

${ }^{10}$ M. L. Yan, R. Skomski, A. Kashyap, L. Gao, S. H. Liou, and D. J. Sellmyer, IEEE Trans. Magn. 40, 2495 (2004).

${ }^{11}$ J. L. Tsai, F. T. Yuan, and S. K. Chen, J. Appl. Phys. 97, 10N122 (2005).

${ }^{12}$ M. L. Yan, R. F. Sabirianov, Y. F. Xu, X. Z. Li, and D. J. Sellmyer, IEEE Trans. Magn. 40, 2470 (2004).

${ }^{13}$ D. H. Ping, M. Ohnuma, K. Hono, M. Watanabe, T. Iwasa, and T. Masumoto, J. Appl. Phys. 90, 4708 (2001).

${ }^{14}$ H. Uwazumi, K. Enomoto, Y. Sakai, S. Takenoiri, T. Oikawa, and S. Watabe, IEEE Trans. Magn. 39, 1914 (2003).

${ }^{15}$ D. Stoyan and J. Mecke, Stochastische Geometrie (Akademieverlag, Berlin, 1983).

${ }^{16}$ J. Weissmüller, A. Michels, J. G. Barker, A. Wiedenmann, U. Erb, and R. D. Shull, Phys. Rev. B 63, 214414 (2001).

${ }^{17}$ J. B. Kortright, O. Hellwig, K. Chesnel, Sh.-H. Sun, and E. E. Fullerton, Phys. Rev. B 71, 012402 (2005).

${ }^{18}$ O. Hellwig, D. T. Margulies, B. Lengsfield, and E. E. Fullerton, and J. B. Kortright, Appl. Phys. Lett. 80, 1234 (2002).

${ }^{19}$ T. A. George, Z. Li, M. L. Yan, Y. F. Xu, R. Skomsi, and D. J. Sellmyer, J. Appl. Phys. 103, 07D502 (2008).

${ }^{20}$ D. J. Sellmyer, Y. F. Xu, Y. C. Sui, and R. Skomski, in Handbook of Magnetism and Advanced Magnetic Materials, edited by H. Kronmüller and S. Parkin (Wiley, New York, 2007), Vol. 4, p. 2177.

${ }^{21}$ J. Zhou, A. Kashyap, Y. Liu, R. Skomski, and D. J. Sellmyer, IEEE Trans. Magn. 40, 2940 (2004).

${ }^{22}$ R. Skomski, J. Phys.: Condens. Matter 15, R841 (2003).

${ }^{23}$ R. Skomski, Simple Models of Magnetism (Oxford University Press, New York, 2008). 